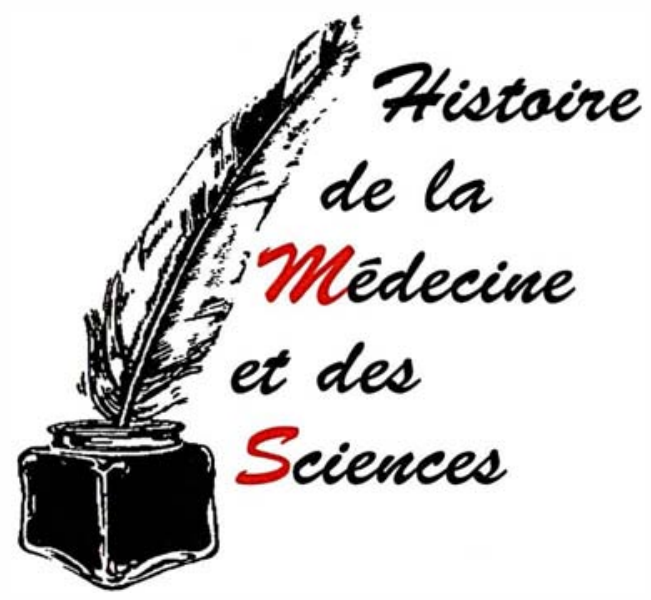

Le sauvage et le criminel

Les criminels et les infracteurs sont-ils biologiquement différents des honnêtes gens?

La plupart des lecteurs de cette rubrique trouveront probablement la question parfaitement ridicule, tant la réponse leur paraît évidente. Et pourtant, il n'en a pas toujours été ainsi. Bien des savants ont postulé cette différence biologique, dès le début du XIX" siècle. L'hypothèse d'une hérédité des vices et des vertus était déjà avancée pour les cas de folie familiale et de criminalité, bien avant que Zola n'en fasse le moteur de l'épopée naturaliste des Rougon-Macquart. Parallèlement à cette montée des thèses héréditaristes, de grands médecins polémiquèrent de 1800 à 1848 sur la théorie phrénologique de François-Joseph Gall (1758-1828), qui postulait que les assassins pouvaient se reconnaître à leur conformation crânienne [1]. Il y a un siècle à peine, beaucoup d'anthropologues, qui étaient pour la plupart de formation médicale, discutaient encore sérieusement du sujet. Les travaux menés sur ce thème connurent leur apogée en Europe entre 1880 et 1914. Durant cette "Belle Époque» de l'anthropologie criminelle, des revues scientifiques spécifiques furent créées, et sept congrès internationaux furent organisés de 1885 à 1911. Les débats de ces congrès virent tout d'abord la domination de l'approche anthropologique mais la théorie de la dégénérescence héritée de B.A. Morel (1809-1873) tendit à la compléter, voire à la remplacer chez

\title{
Les théories biologiques de la criminalité
}

sonnalité scientifique qui domina cette période fut sans conteste le très controversé Cesare Lombroso (18351909), criminologue italien qui popularisa la théorie de l'atavisme criminel (figure 1). S'appuyant sur la préhistoire naissante et sur l'évolutionnisme culturel qui considérait que les sauvages n'avaient pas (ou peu) de morale, Lombroso affirmait que le crime était un fait normal dans le monde animal et dans les sociétés "primitives ". Ainsi, la plupart des crimes commis dans nos sociétés modernes étaient imputables à des individus qui avaient biologiquement et socialement régressé à ces stades antérieurs. La théorie de l'anthropologue italien fourmillait en fait de lieux communs partagés par de nombreux scientifiques de l'époque, qu'ils soient ou non anthropologues. Darwin (1809-1882) lui-même pensait, par exemple, qu'il y avait plus de distance entre l'homme civilisé et le sauvage qu'entre un animal domestique et un animal sauvage [2] et Marcelin Berthelot (1827-1907) écrivait à l'aube de notre siècle que "l'étude des races demeurées sauvages a montré combien leur moralité spéciale était voisine de celle des espèces animales sociables, sinon inférieures pour quelques-unes". Il ne faisait aucun doute pour le célèbre chimiste que "les instincts sociaux, les sentiments et les devoirs qui en dérivent sont [...] inhérents à la constitution cérébrale et physiologique de l'homme" [3].

Si Lombroso avait quelque mal à expliquer l'origine de l'atavisme criminel (il penchait pour un accident dans le développement de l'embryon), il passa en revanche toute sa vie à effec- tuer des mesures comparatives sur les criminels et les honnêtes gens afin de mettre en évidence les caractères anatomiques et physiologiques des infracteurs [4] (Tableau I). Il parvint ainsi à définir un "type-criminel " que l'on reconnaissait à son faciès plus ou moins simiesque, à son insensibilité à la douleur, à son regard, etc. Dans cette théorie du retour atavique des criminels vers le type sauvage, Lombroso achoppa dans un premier temps sur la différence des sexes, car on savait à l'époque (comme maintenant) que les femmes commettent nettement moins de crimes que les hommes. Les femmes seraient-elles donc moins sujettes à l'atavisme que les hommes? Lombroso ne le pensait pas, et il trouva une solution à cette question : il fallait considérer la prostitution féminine comme l'équivalent du crime chez les hommes. Ainsi, Lombroso effectua sur les prostituées les mesures qu'il avait faites sur les criminels et il différencia, là encore, des caractères permettant de reconnaître la véritable "prostituéenée" de la "prostituée d'occasion". Parmi les signes ataviques, plusieurs études avaient montré que la distance entre le gros orteil et le second doigt de pied était en moyenne plus importante chez les prostituées que chez les femmes "normales". Cet écart était, selon les auteurs de ces études, la manifestation d'une certaine aptitude à la préhension du pied de la prostituée, comme celle des singes.

\section{Une théorie dépassée?}

Cette théorie «biologique« de la criminalité apparaît désormais si caricaturale 


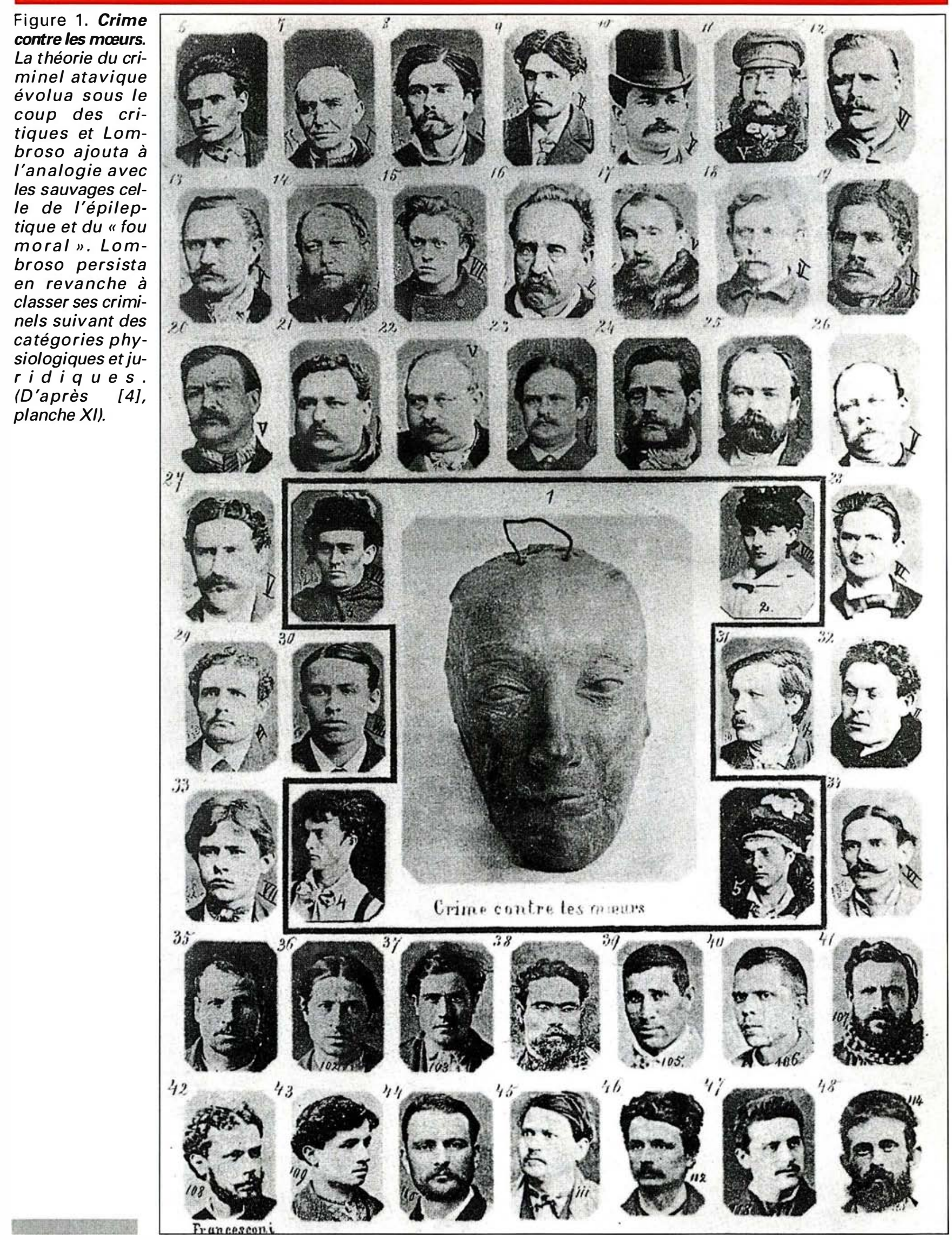




\begin{tabular}{|c|c|c|c|c|c|c|c|c|c|c|c|c|c|}
\hline \multicolumn{14}{|c|}{$\begin{array}{l}\text { Tableau I } \\
\text { ISTINGUER LES CRIMINELS-NÉS (D'après [4], p. 450-1) }\end{array}$} \\
\hline & 薦 & 這 & 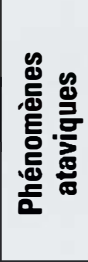 & 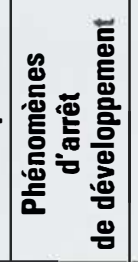 & 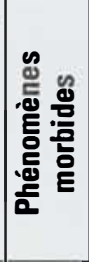 & 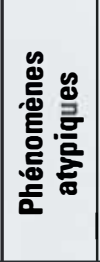 & & 竞 & 兽 & 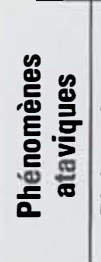 & 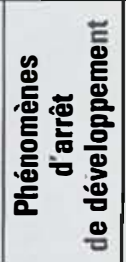 & 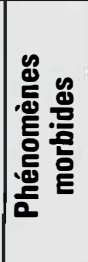 & 㤅 \\
\hline $\begin{array}{l}\text { Crâne } \\
\text { Exagération de volume } \\
\text { Diminution de volume, microcéphalie } \\
\text { Sclérose } \\
\text { Exostose } \\
\text { Asymétrie } \\
\text { Fossette occipitale médiane } \\
\text { lndice crânien exagéré } \\
\text { Arcs sourciliers exagérés } \\
\text { Front bas, fuyant } \\
\text { Front hydrocéphalique } \\
\text { Ostéophites crâniens } \\
\text { Wormiens nombreux } \\
\text { Suture métopique } \\
\text { Sinostoses précoces } \\
\text { Suture crânienne simple } \\
\text { Orbites obliques Face } \\
\text { Appendice lémurien } \\
\text { Mấchoires hypertrophiques } \\
\text { Zygomes saillants volumineux } \\
\text { Diamètre biangulaire de la mấchoire } \\
\text { Oreilles à anses volumineuses } \\
\text { Asymétrie faciale } \\
\text { Strabisme } \\
\text { Physionomie virile chez la femme } \\
\text { Diastème dentaire } \\
\text { Anomalie des os du nez } \\
\text { Anomalies dentaires } \\
\text { Développ. exagéré des os de la face } \\
\text { Anomalie des cerveau } \\
\text { Poids moindrenvol. des scissures } \\
\text { Hypertrophie du cervelet } \\
\text { Attérations histologiques de l'écorce } \\
\text { Traces de méningite } \\
\text { Asymétrie thoracique } \\
\text { Pied préhensile } \\
\text { Gaucherie (Mancinismo) anatomique } \\
\text { Fréquence des lésions viscérales } \\
\text { Hernies } \\
\text { Simplicité des plis palmaires } \\
\text { Thorax à entonnoir } \\
\text { Rides anormales Peau } \\
\text { Rides }\end{array}$ & $\begin{array}{l}+ \\
+ \\
+ \\
+ \\
+ \\
+ \\
+ \\
+ \\
+ \\
+ \\
+ \\
+ \\
+ \\
+ \\
+ \\
+ \\
+ \\
+ \\
+ \\
+ \\
+ \\
+ \\
+ \\
+ \\
+ \\
+ \\
+ \\
+ \\
+ \\
+ \\
+ \\
+ \\
+ \\
+ \\
+ \\
+ \\
+ \\
+ \\
+ \\
+ \\
+\end{array}$ & $\begin{array}{l}+ \\
+ \\
+ \\
+ \\
+ \\
+ \\
+ \\
+ \\
+ \\
+ \\
+ \\
+ \\
+ \\
+ \\
+ \\
+ \\
+ \\
+ \\
+ \\
+ \\
+ \\
+ \\
+ \\
+ \\
+ \\
+ \\
+ \\
+ \\
+ \\
+ \\
+ \\
+ \\
+ \\
+ \\
+ \\
+ \\
+ \\
+ \\
+ \\
+\end{array}$ & $\begin{array}{l}+ \\
+ \\
+ \\
+ \\
+ \\
+ \\
+ \\
+ \\
+ \\
\\
+ \\
+ \\
+ \\
+ \\
+ \\
+ \\
+ \\
+ \\
+\end{array}$ & $\begin{array}{l}+ \\
+ \\
+ \\
+ \\
+ \\
+ \\
+ \\
+\end{array}$ & $\begin{array}{l}+ \\
+ \\
+ \\
+ \\
+ \\
+\end{array}$ & + & 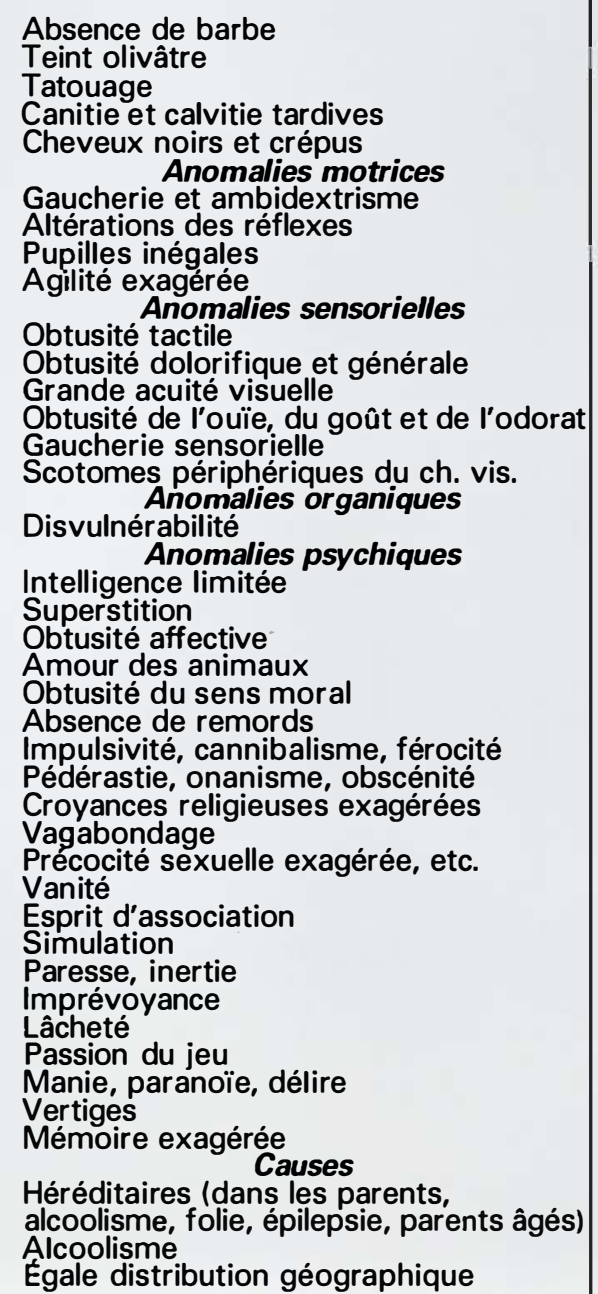 & $\begin{array}{l}+ \\
+ \\
+ \\
+ \\
+ \\
+ \\
+ \\
+ \\
+ \\
+ \\
+ \\
+ \\
+ \\
+ \\
+ \\
+ \\
+ \\
+ \\
+ \\
+ \\
+ \\
+ \\
+ \\
+ \\
+ \\
+ \\
+ \\
+ \\
+ \\
+ \\
+ \\
+ \\
+ \\
+ \\
+ \\
+ \\
+\end{array}$ & $\begin{array}{l}+ \\
+ \\
+ \\
+ \\
+ \\
+ \\
+ \\
+ \\
+ \\
+ \\
+ \\
+ \\
+ \\
+ \\
+ \\
+ \\
+ \\
+ \\
+ \\
+ \\
+ \\
+ \\
+ \\
+ \\
+ \\
+ \\
+ \\
+ \\
+ \\
+ \\
+ \\
+ \\
+ \\
+ \\
+ \\
+ \\
+ \\
+ \\
+\end{array}$ & $\begin{array}{l}+ \\
+ \\
+ \\
+ \\
+ \\
+ \\
+ \\
+ \\
+ \\
+ \\
+ \\
+ \\
+ \\
+ \\
+ \\
+ \\
+ \\
+ \\
+ \\
+ \\
+ \\
+ \\
+ \\
+ \\
+ \\
+ \\
+\end{array}$ & + & $\begin{array}{l}+ \\
+ \\
+ \\
+ \\
+ \\
+ \\
+\end{array}$ & \\
\hline
\end{tabular}

Ce tableau donne une bonne idée du souci d'exhaustivité propre aux anthropologues positivistes. On y remarque que les " anomalies psychiques" complètent les indices biologiques. Le seul critère qui ne soit pas commun aux "criminels-nes"s et aux "épileptiques" est le tatouage. Notons aussi que tous les stigmates n'étaient pas systématiquement imputés au phénomène d'atavisme 
qu'elle ne peut nous laisser indifférent. A-t-elle seulement sa place dans une rubrique d'histoire des sciences? Peut-on considérer Lombroso comme un savant, alors que ses travaux accumulent des biais statistiques importants? C'est dans ces questions, épistémologiques autant qu'historiques, que se joue l'actualité de la théorie du criminel-né. Son intérêt didactique n'est pas négligeable, tant sur le plan pédagogique que sur celui de notre réflexion sur la place des discours scientifiques dans nos sociétés. L'aspect pédagogique est évident, car les erreurs de Lombroso sont si flagrantes qu'elles sont une bonne illustration des pièges les plus simples que revêt l'outil statistique (échantillonnage non représentatif, corrélation prise comme cause, erreur d'interprétation) [5]. Il faudrait se garder pourtant d'en déduire que Lombroso était un mauvais scientifique. Ses adversaires lui opposèrent dans la controverse des arguments ou des propositions alternatives qui nous paraîtraient tout aussi irrecevables aujourd'hui [6]. Alexandre Lacassagne (1843-1924), par exemple, chef de file dans les années 1880 de l'opposition des Français à Lombroso, pensait que les criminels pouvaient se diviser selon leur activité cérébrale : il distinguait ainsi des "occipitaux ", des "pariétaux " et des "frontaux" et les types de peines devaient être ajustés à leur caractère.

Le paradoxe dans cette question, c'est queles théories « scientifiques » qui ont le plus d'impact socialement sont aussi celles dont les auteurs défendent l'image d'une science pure, objective et apolitique. Lombroso aimait répéter qu'il était "esclave des faits" et qu'il travaillait sans a priori. Discours indispensable pour convaincre le public de la légitimité et du bien-fondé de son propos. On ne s'étonnera pas que ce mythe d'une science pure ait été construit par les positivistes du XIX siècle (Auguste Comte), ceux-là même qui pensaient que "la science" devait prendre la place de la religion dans une société vraiment rationnelle. Si l'anthropologie criminelle tenta de se constituer comme discipline autonome autour des débats sur la théorie lombrosienne dans les années 1880, la recherche des caractères biologiques des criminels débuta en fait en Europe $\mathrm{m} / \mathrm{s} n^{\circ} 12$, vol. 11, décembre 95 occidentale dès le début du XIX ${ }^{\mathrm{e}}$ siècle. Elle s'inscrivait dans une volonté de réduction médicale des déviances (folie, assassinat, suicide, vols, perversions sexuelles, etc.) qui n'est pas totalement abandonnée de nos jours. Loin de rester dans leur cabinet de consultation, hôpital ou laboratoire, la plupart des médecins du XIX ${ }^{\mathrm{e}}$ siècle voulaient agir sur la société. L'hygiénisme, les vaccinations et la médecine sociale font partie du bilan positif de cet engagement. Mais quelques-uns allèrent plus loin. Postulant que le physique et le moral étaient en étroite relation, ils tentèrent d'expliquer les déviances sociales par des raisons physiques. La plupart des déviances apparemment incompréhensibles (assassinats et vols sans mobiles en particulier) devenaient potentiellement explicables par la dégénérescence physique et morale des individus (monomanie homicide, pyromanie, kleptomanie...). Ce savoir permit aux aliénistes d'entrer dans le prétoire comme médecins-experts pour évaluer la santé mentale des accusés. Ils furent souvent à cette occasion en conflit avec les juristes, qui fondent le droit de punir sur la responsabilité des individus. Que devait-on faire des individus auxquels on ne pouvait imputer le crime, qui étaient inaccessibles à toute intimidation et qui s'avéraient dangereux? Passant dans un premier temps pour les "amis des prisonniers ", les médecins de la seconde moitié du XIX ${ }^{\mathrm{e}}$ siècle défendirent une théorie pénale alternative, "positiviste", qui proposait de condamner, non plus en fonction de la gravité de l'acte commis, mais de la dangerosité sociale de l'individu. L'idéal de certains médecins était alors la sentence d'enfermement à durée indéterminée, l'individu ne devant sortir que lorsqu'il serait "guéri". Cette peine n'existait pas en France, mais elle fut appliquée dans quelques pays, dont les Etats-Unis. Notre système pénal oscille désormais entre ces deux tendances, comme le montrent les mesures législatives prises en France en 1993 sous la pression des crimes de pédophiles.

\section{L'actualité du passé}

L'intérêt de garder en mémoire la théorie de Lombroso, et plus largement l'anthropologie criminelle qu'elle in- carne, est que l'aporie de cette tentation médicalisante persiste malgré les réfutations successives. L'affaire du chromosome du crime, qui fit grand bruit dans les années 1960, en est une bonne illustration, tout comme les expériences de psychochirurgie qui furent menées aux États-Unis dans la décennie suivante [7]. L'échec du chromosome XYY n'a pas clos le débat sur le terrain génétique et l'on évoquait encore récemment une détermination génétique de l'homosexualité. Ces recherches génétiques sont, qu'elles le veuillent ou non, indissolublement liées au spectre de l'eugénisme [8]. Ce n'est évidemment pas à l'historien des sciences de décider si telle ou telle catégorie d'infraction grave ou de déviance implicite recouvre un domaine pathologique précis. Ce qu'il peut apporter dans la réflexion, en revanche, c'est que les fréquentes erreurs que l'on observe dans le passé sont produites par la volonté de normaliser certaines catégories normatives construites par le droit. Cette substitution d'un discours scientifique à un discours juridique a pour effet pernicieux de naturaliser le fonctionnement effectif de l'appareil juridique, qui est souvent en décalage avec le droit des codes. L'hypothèse d'anormalité biologique retombe souvent sur les mêmes catégories sociales. On n'a jamais vu, en effet, un médecin ou un biologiste chercher le type anatomique ou le gène du politicien corrompu alors que des théories sur les infériorités biologiques des pauvres ou des "marginaux" n'ont cessé d'être avancées depuis bientôt deux siècles.

Le fait que l'on reproduise ainsi les mêmes erreurs à plusieurs années d'intervalle doit nous inciter à réfléchir à la fois au statut de ces théories par rapport à l'histoire de nos disciplines et à l'engagement des scientifiques dans leur temps. Les scientifiques ont-ils droit à l'erreur? Quelle est la différence entre un scientifique qui se trompe et un pseudo-scientifique? Les théories "fausses" doiventelles être conservées dans l'histoire de la médecine par exemple? Quel que soit le statut que l'on accorde à ces discours, "fausse science", "erreur", "idéologie", nous aurions probablement beaucoup à perdre à sous-estimer leurs effets dans nos sociétés. Il ne 
suffit pas, malheureusement, qu'une théorie soit erronée ou dangereuse (ce qui n'est pas toujours la même chose) pour qu'elle n'ait pas de succès [9]. L'histoire des sciences montre ainsi que la logique de la diffusion des théories à prétention scientifique n'a qu'un rapport faible avec leur rationalité ou, plutôt, que les raisons de diffusion de telle ou telle théorie sont multiples, et obéissent autant à des intérêts sociaux, économiques et politiques que purement scientifiques. La reconnaissance de ce fait, loin de déconsidérer la valeur des savoirs scientifiques, peut donner des arguments à tous ceux qui n'entendent pas rester indifférents devant certains usages politiques des sciences

\section{RÉFÉRENCES}

1. Lantéri-Laura G. Histoire de la phrénologie (l'homme et le cerveau selon F.J. Gall). Paris : PUF, 1993.

2. Darwin CR. Voyage d'un naturaliste autour du monde. Tome I (Des îles du Cap-Vert à la terre de Feu). Paris : François Maspero, 1982: 228.

3. Berthelot M. Science et morale. Paris: Calmann-Lévy, 1897: 25.

4. Lombroso C. L'homme criminel (criminel né fou moral-épileptique). Paris: Félix Alcan, 1887.

5. Gould SJ. La mal-mesure de l'homme. Paris: Ramsay, 1983: 153-7.

6. Mucchielli L. Histoire de la criminologie française. Paris: L'Harmattan, 1995.

7. Valenstein SE. The psychosurgery debate (scientific legal and ethical perspectives). San Francisco: W.H. Freeman and Cie, 1980.

8. Taylor L. Born to crime (the genetic causes of criminal behavior). Londres: Greenwood Press, 1984.

9. Duster T. Retour à l'eugénisme. Paris: Kimé, 1992.

\section{Remerciements}

Je remercie Claude Schnitter pour ses suggestions et sa disponibilité.

Marc Renneville

Université Paris VII, U.F. Anthropologie, 10, rue Charles-V, 75004 Paris, France.

\section{TIRÉS À PART}

M. Renneville. 